\title{
HABILIDADES SOCIAIS E ABUSO DE DROGAS EM ADOLESCENTES ${ }^{1}$
}

\author{
Marcia Fortes Wagner* \\ Margareth da Silva Oliveira**
}

\section{RESUMO}

O presente estudo propõe uma revisão bibliográfica sobre habilidades sociais e abuso de substâncias. Este artigo foi elaborado a partir de pesquisa nas Bases de Dados Pschynfo, Web of Science, Cochrane Library, Proquest, Medline e Lilacs, entre 1996 e 2006. Os descritores foram: social skills, social skills training, social competence, assertiveness, adolescents, teeenagers, substance abuse, drug abuse, cannabis e marijuana. Nas bases de língua portuguesa, foram: habilidades sociais, treinamento em habilidades sociais, assertividade, adolescentes, abuso de substâncias, drogas e maconha. Os estudos, a maioria de língua inglesa, apontaram a existência de déficits, principalmente a dificuldade em resistir às drogas e dizer não. Conclui-se que a construção de habilidades de resistência ao oferecimento de drogas, a autoeficácia e o estímulo à capacidade de tomada de decisões pode reduzir o uso de substâncias. Poucos estudos brasileiros foram encontrados abordando esta temática.

Palavras-chave: habilidades sociais, adolescência, abuso de substâncias, maconha

\section{Abstract}

SOCIAL SKILLS AND DRUG ABUSE IN ADOLESCENTS

The present article proposes a bibliographic revision on social skills and drug abuse. This article was elaborated from a research in the following Databases: Pschynfo, Web of Science,

* Mestre em Psicologia Clínica; Doutoranda em Psicologia pela Pontifícia Universidade Católica do Rio Grande do Sul; Psicóloga Clínica e Professora do Curso de Psicologia da Faculdade Meridional/IMED, Passo Fundo, Rio Grande do Sul.

** Doutora em Ciências pela Universidade Federal de São Paulo; Professora da Faculdade de Psicologia e do Programa de Pós-Graduação em Psicologia da Pontifícia Universidade Católica do Rio Grande do Sul. 
Cochrane Library, Proquest, Medline and Lilacs, between 1996 and 2006. The used describers were: social skills, social skills training, social competence, assertiveness, adolescents, teenagers, substance abuse, drug abuse, cannabis and marijuana. In the databases in Portuguese language we used the terms habilidades sociais, treinamento em habilidades sociais, assertividade, adolescentes, abuso de substancias, drogas and maconha. The studies, the majority of which were in English, pointed the existence of deficits, mainly the difficulty in resisting to drugs and in saying no. It can be concluded that the construction of resistance skills to the offering of drugs, selfefficacy training and the stimulation of decision making capabilities can reduce substance use. Few Brazilian studies were found approaching this subject.

Keywords: social skills, adolescents, substance abuse, marijuana

\section{INTRODUÇÃO}

A adolescência é um período de transição entre a infância e a vida adulta caracterizado pela necessidade de integração social, busca da auto-afirmação e da independência individual, além da consolidação da identidade sexual e emoções conflitantes (Silva \& Mattos, 2004). Pode ser considerada como uma etapa do desenvolvimento que envolve inúmeras adaptações e mudanças nas capacidades e habilidades pessoais.

Alguns estudos referem que, por estar em um momento de maior vulnerabilidade, no qual podem estar presentes sentimentos de insegurança e desamparo frente às mudanças físicas e psicológicas próprias desta etapa do ciclo vital, o adolescente ainda não desenvolveu de forma adequada algumas habilidades e demonstra a constante necessidade de testar sua possibilidade de ser adulto (Nightingale \& Fischhoff, 2002). Em busca de poder e controle sobre si mesmo, adquirir autonomia e diferenciar-se de seus pais, alguns jovens escolhem usar drogas, geralmente iniciando com cigarros, álcool, maconha, o que pode levar, conseqüentemente, ao uso de múltiplas drogas ilícitas (Bolognini, Plancherel, Laget \& Halfon, 2004; Kaminer \& Szobot, 2004; Marques \& Cruz, 2000).

Os dados do Centro Brasileiro de Informação Sobre Álcool e Outras Drogas CEBRID constataram um aumento no consumo de substâncias psicoativas na grande maioria das cidades brasileiras. Da população estudada, 19,4\% já fez uso de drogas na vida. Em relação aos estudantes na faixa etária entre 12 a 17 anos, $3,5 \%$ elegeram a maconha como a droga de escolha. Nos jovens de 18 a 24 anos, 9,9\% confirmaram o uso da maconha, sendo que $1 \%$ da população deste estudo foi considerada dependente, correspondendo a um total de 451.000 pessoas (Carlini, Galduróz, Noto \& Nappo, 2001). 
Entre as drogas ilícitas, a maconha é a mais usada no Brasil. Como ocorre com a maioria das outras drogas ilícitas, a maconha é mais freqüentemente utilizada por homens (American Psychiatric Association, APA, 2002). Conforme Noto (2004), em levantamento realizado em 1997, 7,6\% dos estudantes relataram já ter experimentado maconha ao menos uma vez na vida. As capitais que mais apresentaram consumo estão na região Sul, sendo 11,9\% em Curitiba e 14,4\% em Porto Alegre. Tavares, Béria e Lima (2001) confirmaram estes dados num estudo de prevalência do uso de drogas entre adolescentes de escolas de Ensino Médio, constatando que, entre as drogas ilícitas usadas, a maconha também apareceu em primeiro lugar e com maior uso por meninos.

Algumas contribuições atuais à área dos transtornos associados ao uso de substâncias estão sendo desenvolvidas através de pesquisas que buscam comprovar a relação entre a dependência de substâncias psicoativas e a existência de déficits nas habilidades sociais dos indivíduos usuários ou abusadores de drogas. Como refere Graña Gómez (2001), os fatores de risco e proteção para o consumo de drogas em adolescentes incluem fatores psicológicos e influências do grupo de pares. Levando em consideração tais aspectos, é necessário que os jovens aprendam a manejar suas características de caráter psicológico que os exponham a uma situação de risco, potencializando aquelas que possam protegê-los frente ao início do consumo de substâncias psicoativas.

Esse modelo dos déficits nas habilidades sociais apóia a hipótese de que as crianças que não desenvolveram precocemente habilidades de interagir socialmente de uma forma adequada podem ser rechaçadas por seus pares e acabar se envolvendo em comportamentos pouco saudáveis como, por exemplo, violência e uso de drogas ilícitas. Numa perspectiva do desenvolvimento da saúde mental, os programas preventivos focalizam-se no treinamento assertivo e nas estratégias de comunicação para o rechaço e a negociação frente às drogas, em combinação com habilidades para solução de problemas e tomada de decisões (Organización Panamericana de la Salud, 2001).

Habilidades sociais ou comportamento socialmente habilidoso pode ser definido como:

um conjunto de comportamentos emitidos por um indivíduo em um contexto interpessoal que expressa sentimentos, atitudes, desejos, opiniōes ou direitos desse indivíduo de modo adequado à situação, respeitando esses comportamentos nos demais, e que geralmente resolve os problemas imediatos da situação enquanto minimiza a probabilidade de futuros problemas (Caballo, 1998: 6). 
Outra conceituação propõe que:

as habilidades sociais correspondem a um universo mais abrangente das relaçôes interpessoais e se estendem para além da assertividade, incluindo as habilidades de comunicação, de resolução de problemas, de cooperação e aquelas próprias dos rituais sociais estabelecidos pela subcultura grupal (Del Prette \& Del Prette, 1999: 29).

Oliveira (2002) afirma que o aprendizado de novas habilidades interpessoais capacita os indivíduos que possuem dificuldades para serem assertivos a defenderem seus direitos de forma mais efetiva quando houver a pressão de outras pessoas para consumirem drogas. Nesse contexto, o tratamento a partir do treinamento de habilidades sociais pode, então, auxiliar na recuperação das lacunas existentes, através da instrumentalização do jovem com um leque de comportamentos mais saudáveis.

Treinamento em Habilidades Sociais (THS), de acordo com Caballo (2003), pode ser compreendido como um tratamento que visa ensinar estratégias e habilidades interpessoais aos indivíduos, com a intenção de melhorar a sua competência interpessoal e individual em classes específicas de situações sociais. O THS vem sendo considerado como auxiliar no tratamento do abuso e dependência de substâncias psicoativas, contemplando a promoção de habilidades sociais dos dependentes, além da criação e maximização das redes de apoio social (família, trabalho e religião), buscando a prevenção dos comportamentos dependentes e das recaídas de ex-dependentes (Caballo, 2003; Del Prette \& Del Prette, 1999).

O presente artigo analisa a literatura relacionada ao papel das habilidades sociais no contexto dos adolescentes usuários de drogas ilícitas, em especial a maconha, considerando que, na prática clínica, constata-se que muitos indivíduos acabam buscando no uso de substâncias psicoativas uma forma de se tornarem mais sociáveis e com melhor capacidade de interação com seus pares. Nesse sentido, além da dificuldade do adolescente em dizer não ao grupo de iguais, o uso de drogas pode produzir um reforçador social, além de reduzir sintomas de ansiedade decorrentes de contingências aversivas, os quais poderiam ser reduzidos e/ou suprimidos se outras habilidades sociais fossem promovidas nos diferentes contextos nos quais o jovem está inserido, tais como família, escola, amigos e relacionamentos amorosos.

\section{MéTodo}

Este é um artigo de revisão bibliográfica elaborado a partir de uma pesquisa nas bases de dados Cochrane Library, Lilacs, Medline, Proquest, PsycINFO e Web of 
Science, no intervalo dos anos de 1996 a 2006. Os descritores utilizados foram social skills, social skills training, social competence, assertiveness, adolescents, teeenagers, substance abuse, drug abuse, cannabis e marijuana. Os descritores nas bases de língua portuguesa foram habilidades sociais, treinamento em habilidades sociais, assertividade, adolescentes, abuso de substâncias, drogas e maconha. Também foram analisados livros e artigos que não se encontravam nas referências das fontes indexadas.

A busca inicial resultou em 223 resumos, levando-se em consideração os descritores e a data escolhidos. Realizada uma primeira leitura e constatada a necessidade de descartar aqueles que abordavam habilidades sociais em alguns transtornos, como, por exemplo, na esquizofrenia, na deficiência mental, no transtorno obsessivo-compulsivo, entre outros, a busca foi refinada e obteve-se uma redução de 223 para 106 artigos.

Após leitura criteriosa, descartaram-se artigos que abordavam o tema de forma geral em outras áreas ou relacionado ao treinamento de habilidades sociais de pais, professores e crianças. Procedeu-se à seleção dos 49 artigos finais dessa revisão, com ênfase nos aspectos teóricos, na avaliação das habilidades sociais no uso de substâncias e nos programas de treinamento de habilidades sociais. A seleção dos 49 artigos nas bases de dados é ilustrada na Tabela 1 (ver Anexo).

\section{APRESENTAÇÃO E DisCUSSÃO DOS ARTIGOS}

Nos trabalhos publicados nos últimos dez anos, vem sendo produzido um consistente referencial sobre habilidades sociais, voltadas à avaliação e à intervenção. A avaliação busca identificar os déficits e reaçôes emocionais disfuncionais que interferem na expressão do comportamento habilidoso (Del Prette \& Del Prette, 1999), com a utilização de alguns instrumentos como entrevistas, inventários, auto-registros e observação do comportamento, os quais podem ser aplicados ao próprio sujeito, aos pais ou aos professores (Caballo, 2003; Del Prette \& Del Prette, 1999; Falcone, 2002). Já a etapa da intervenção está relacionada ao treinamento em habilidades sociais, com utilização de inúmeras técnicas cognitivocomportamentais, como fornecimento de instruções, ensaio comportamental, modelagem, tarefas de casa, feedback verbal e em vídeo, reestruturação cognitiva, solução de problemas, relaxamento e vivências grupais (Caballo, 2003).

Dentre os 49 artigos selecionados, 16 referem-se à avaliação das habilidades sociais, enquanto 20 apresentam ênfase nos programas de intervenção relacionados à mudança comportamental voltada à prevenção, à redução ou à cessação do uso de substâncias psicoativas, através do treinamento de habilidades. Os demais 
13 artigos referem-se aos aspectos teóricos do tema habilidades sociais no contexto de indivíduos que fazem uso de substâncias, tais como estudos de revisão sobre competência social, assertividade, déficits em habilidades sociais na adolescência e o uso de drogas.

Segundo Bolsoni-Silva (2002), o estudo teórico e prático do THS é fundamental, visto que os indivíduos ficam muito tempo engajados em interações sociais. Ao conseguirem ser socialmente habilidosos, promovem interaçôes sociais satisfatórias, com aumento dos reforçadores, o que pode auxiliar na prevenção el ou redução de dificuldades psicológicas. Alguns estudos de habilidades sociais em população de universitários, numa amostra não clínica, foram desenvolvidos por Furtado, Falcone \& Clark (2003) e Del Prette, Del Prette, Barreto, Bandeira, Rios-Saldaña, Ulian, Gerk-Carneiro, Falcone \& Vila (2004), os quais concluem que deficiências nessas habilidades podem contribuir para o desenvolvimento do estresse.

Já há na literatura fortes evidências das relações entre as habilidades sociais e os transtornos psicológicos, entre eles esquizofrenia, depressão, transtornos emocionais da infância e adolescência, transtornos afetivos e de ansiedade em qualquer etapa da vida, transtornos invasivos, como autismo, e no abuso e dependência de substâncias psicoativas (Del Prette \& Del Prette, 2002; Falcone, 2000). No caso específico dos transtornos por uso de substâncias, os chamados déficits em habilidades sociais podem estar presentes sob a forma de baixa competência social e dificuldades específicas, como enfrentamento de situações de risco à autoestima e resolução de problemas. Essas dificuldades levam o jovem a uma fuga, via uso de substâncias, as quais ocasionam ainda mais perturbaçóes em seu desempenho social, além de que a pressão do grupo de pares pelo uso da droga exige um comportamento assertivo de saber recusar (Scheier, Botvin, Diaz \& Griffin, 1999).

Dessa forma, é possível afirmar que problemas em diferentes áreas do funcionamento diário do indivíduo são fortemente relacionados ao consumo de álcool e outras drogas entre os jovens (Kaminer, 1999). Algumas publicaçôes fazem referência ao fato de que os adolescentes estão progressivamente aumentando o consumo de drogas psicoativas, levando ao abuso e dependência dessas substâncias cada vez mais em idade precoce. Estudos de Kaminer (1999), Tripathi, Lal \& Kumar (2001) e Simkin (2002) confirmam esses dados, referindo que entre as substâncias mais comumente utilizadas estão o álcool, o tabaco e a maconha.

Botvin, Malgady, Griffin, Scheier \& Epstein (1998) fazem referência aos fatores sociais de risco que, associados ao uso de substâncias psicoativas, tais como o álcool e a maconha, estão estreitamente relacionados no caso de adolescentes que apresentam habilidades interpessoais empobrecidas. Outros autores referem 
também a existência de predisposição biológica, habilidades sociais inadequadas, rejeição social, baixa qualidade e pouca consistência das relações familiares, problemas emocionais e psiquiátricos, história de comportamento anti-social e delinqüência (Spooner, 2000; Weinberg, 2001), além de baixa auto-estima e déficits precoces no funcionamento psicológico (Scheier, 2001), relacionando tais fatores ao abuso de substâncias.

Barkin, Smith \& Durant (2002) realizaram uma investigação com 2646 alunos, na Universidade de Wake Forrest, Carolina do Norte, com o objetivo de examinar como as atitudes e as habilidades sociais dos adolescentes podem influenciar o uso de substâncias e as intençôes de fazer tal uso no futuro. Os achados comprovam a existência de déficits, principalmente a dificuldade em resistir às drogas e dizer não, mas também concluem que a construção de habilidades de resistência ao oferecimento de drogas e de auto-eficácia, além do estímulo à capacidade de tomada de decisões, pode reduzir o comportamento de uso dessas substâncias.

Cada vez mais estudiosos estão despertando o seu interesse por estratégias preventivas ao uso de substâncias, baseadas em um enfoque psicossocial, o que se confirma nas publicações envolvendo o assunto no meio científico. Nessa perspectiva, percebe-se que as mais atualizadas abordam o desenvolvimento de habilidades sociais e, mais especificamente, de habilidades de recusa às drogas, como uma das formas de prevenção. Segundo Lörh (2001), uma intervenção preventiva deve facilitar a aprendizagem de novas habilidades sociais e prevenir a redução de comportamentos inadequados. Isso pode auxiliar as crianças a buscarem desde cedo maneiras mais assertivas de relacionamento com seus pares, o que os tornará adultos que saberão lidar melhor com as dificuldades que surgirem na vida. Sendo assim, programas preventivos devem focalizar o desenvolvimento da assertividade, bem como da empatia e da solução de problemas (Falcone, 2000, 2002), visando aumentar as competências sociais.

Nas revisões de literatura mais atualizadas, destaca-se a importância das intervenções psicoterápicas no abuso e dependência de maconha na adolescência (Denis, Lávie, Fatséas \& Auriacombe, 2006; Kaminer, 1999; Williams \& Chang, 2000). Algumas fazem um estudo comparativo entre os diferentes tipos de intervenções, como a entrevista motivacional ou intervenção breve, treinamento de habilidades sociais, intervençôes familiares e comunitárias (Gates, McCambridge, Smith \& Foxcroft, 2005). Os achados evidenciam que os resultados de um tratamento são superiores ao não tratamento, ou seja, a maioria dos adolescentes que recebeu tratamento apresentou reduções significativas no uso de substâncias e nos problemas em outras áreas da vida no ano subseqüente ao tratamento. Entretanto, 
não foram encontradas evidências suficientes para comprovar a efetividade entre os diferentes tipos de tratamentos.

Outro estudo realizado por Botvin, Epstein, Baker, Diaz \& IfillWilliams (1997) testou a efetividade de uma intervenção preventiva ao abuso de drogas, utilizando uma amostra de 721 escolares urbanos de uma população minoritária americana, na qual eram ensinadas habilidades de resistência social, em um contexto de ampla intervenção voltada à promoção da competência pessoal e social, atividade implementada por professores em sala de aula. Os resultados indicaram que a abordagem foi efetiva em vários comportamentos relacionados ao uso de drogas, incluindo o uso de múltiplas drogas e nas medidas de intenção de uso futuro.

Diversos artigos encontrados abordam a influência positiva do desenvolvimento das habilidades sociais como fator preventivo ao uso de substâncias (Botvin, 2000a; Botvin \& Griffin, 2004; Goldberg-Lillehoj, Spoth \& Trudeau, 2005). Nesse sentido, Eisen, Zellmann \& Murray (2003) investigaram uma amostra de 7426 adolescentes, em 34 escolas, e também concluíram que programas preventivos ao uso de drogas incrementam a auto-eficácia perante a recusa às drogas. Confirmando essa idéia, estudos de revisão de Botvin e Griffin (2002, 2004) e Faggiano, Vigna-Tagliant, Versino, Zambon, Borraccino \& Lemma (2006) sobre os efeitos de programas preventivos para adolescentes abusadores de drogas durante a adolescência, com foco na identificação dos fatores de risco e proteção associados ao início do uso de drogas, comprovaram o efeito positivo da instrumentalização do jovem através do ensino de habilidades relacionadas à resistência social ou dizer não às drogas e aumento da competência pessoal e social.

Reforçando esse efeito positivo, Amengual (2000) já havia analisado revisōes de literatura, bem como diversos programas e intervençôes. Os resultados evidenciaram que os programas mais eficazes são os que não se centram somente no uso da maconha, e sim aqueles que apontam os diversos componentes da conduta de usar drogas. Além disso, os programas que centram sua atenção exclusivamente na transmissão de informação não reduzem o consumo de drogas, nem as práticas de risco.

Trudeau, Lillehoj, Spoth \& Redmond (2003) pesquisaram os processos de mediação entre a assertividade e a tomada de decisão no início precoce do uso de substância numa amostra de 357 adolescentes. Os resultados também indicaram que os efeitos individuais da assertividade e da tomada de decisão têm relação indireta no início do uso, bem como nas expectativas e na recusa das drogas. Nos meninos, foi constatado que a iniciação precoce do uso de substância foi associada negativamente com níveis mais tardios de expectativas negativas e intençóes de recusa. 
Constatou-se neste estudo um predomínio de publicações de língua inglesa, com uma pequena produção nacional sobre habilidades sociais nos transtornos por uso de substâncias. Murta (2005) faz uma revisão de literatura sobre a produção brasileira envolvendo programas de treinamento em habilidades sociais. Conclui que a publicação atual sobre o assunto em nosso país apresenta início recente, mas com delineamentos pré-experimentais em contextos diversificados e com cuidados metodológicos relevantes.

Foi possível apurar que alguns programas americanos e espanhóis vêm sendo implementados já há alguns anos. Tais programas ensinam aos jovens habilidades para a vida, como resistir às influências sociais para o consumo de drogas e estimular o desenvolvimento de habilidades interpessoais (Amengual, 2000; Botvin, 2000a; Botvin \& Kantor, 2000; Eisen, Zellmann \& Murray, 2003; Scheier \& Botvin, 1998).

Um programa chamado Habilidades de Vida, voltado a adolescentes e professores, foi desenvolvido por Gorayeb, Cunha Netto \& Bugliani (2003) no Brasil, com o objetivo de desenvolver habilidades para lidar com diversas situaçóes de risco, entre as quais o uso de drogas. Através de sessôes grupais, ocorreu o treino em habilidades de decisão, resolução de problemas, pensamento crítico, pensamento criativo, comunicação eficaz, relacionamento interpessoal, autoconhecimento, empatia, lidar com as emoções e com o estresse. Os resultados evidenciaram que os adolescentes apresentaram melhoras na interação grupal, na interação com o facilitador do grupo, nas relaçôes interpessoais fora do grupo, além de aumento de consciência sobre as situações de risco e as habilidades necessárias para manejá-las de forma adequada. Minto, Pedro, Netto, Bugliani \& Gorayeb (2006) analisam os temas, conteúdos e metodologias adotadas nessa intervenção, destacando sua importância como uma estratégia para tornar os adolescentes mais competentes para enfrentar as demandas de sua vida cotidiana.

Diversos estudos foram encontrados, com o foco nos programas preventivos ao uso de substâncias, baseados no treinamento de habilidades sociais de recusa e de resistência às drogas (Donohue, Van-Hasselt, Vincent, Hersen \& Perrin, 1999; Herrmann \& McWhirter, 1997; Rosenbaum \& Hanson, 1998). Muitos consistem em programas integrantes do currículo escolar, desenvolvidos ao longo do ensino médio durante dois ou três anos (Botvin, 2000b; Hecht e cols., 2003; O'Hearn \& Gatz, 2002; Payton, Wardlaw, Graczyk \& Bloodworth, 2000).

Um desses programas preventivos ao abuso de drogas, aplicado a 3.621 estudantes de grupos minoritários em Nova Iorque, foi relatado por Botvin, Griffin, Diaz \& Ifill-Williams (2001). Foi estimulado o desenvolvimento de habilidades de recusa frente às drogas, normas antidrogas, habilidades pessoais de autodesen- 
volvimento e habilidades sociais gerais. Tal programa tinha o objetivo de instrumentalizar o jovem com habilidades e conhecimentos para conseguir resistir ao oferecimento das drogas, diminuir a motivação para o uso e a vulnerabilidade para as influências sociais do uso de drogas. Os resultados indicaram que os 2.144 sujeitos do grupo experimental que receberam o programa relataram diminuição do uso de substâncias psicoativas, em relação aos 1.477 sujeitos do grupo controle que não receberam o programa, permitindo concluir que os programas de desenvolvimento de habilidades sociais podem ser eficazes na prevenção ao uso de substâncias. Brandt (2003) corrobora esses resultados em sua investigação de programas de prevenção ao abuso de substâncias em adolescentes da África do Sul, na qual os achados confirmaram que o desenvolvimento de habilidades sociais e pessoais foi efetivo na prevenção ao uso de drogas, com diferença significativa nas mudanças de atitudes entre os usuários.

Nessa mesma direção, Alexandre, Del Rio \& Pol (2004) realizaram uma investigação com 314 jovens entre 18 a 30 anos, com o objetivo de analisar o valor das estratégias de enfrentamento, habilidades sociais e habilidades individuais no consumo de diferentes substâncias. Concluiu-se que déficits em habilidades sociais e do próprio indivíduo influenciam no consumo de álcool e maconha, e a modificação desses déficits pode permitir a realização de programas de prevenção mais efetivos.

Por outro lado, Aliane, Lourenço \& Ronzani (2006) fizeram um estudo comparativo das habilidades sociais de dependentes e não dependentes de álcool no Brasil, em uma amostra de 80 sujeitos. Os achados revelaram que, apesar de a literatura sobre o tema discutir os déficits de HS entre dependentes, não foram encontradas diferenças estatísticas significativas entre os grupos. Suelves \& SánchezTuret (2001) realizaram um estudo transversal sobre o treinamento da assertividade em programas de prevenção ao abuso de substâncias com 294 adolescentes espanhóis, no qual as conclusōes também relataram uma ausência de provas claras da relação entre assertividade e uso de substâncias psicoativas. Porém o fator agressividade apresentou correlaçôes estatisticamente significativas com o uso de tabaco, álcool e maconha. Tais dados justificam a necessidade de novas pesquisas a respeito das habilidades assertivas no início do consumo de substâncias.

\section{CONSIDERAÇÕES}

A literatura revisada mostrou fortes evidências de que adolescentes abusadores e dependentes de substâncias psicoativas, em especial a maconha, podem apresentar déficits nas habilidades sociais. Del Prette \& Del Prette (2002) e Falcone (2000) 
fazem referência a esses déficits nas conclusões de estudos desenvolvidos sobre o assunto. Outra constatação é que, além dos fatores psicológicos, existe a influência do grupo de pares, como refere Graña Gómez (2001). Nesse sentido, para ser aceito pelo grupo de iguais, o jovem pode sentir-se pressionado a ter o mesmo comportamento de uso de substâncias psicoativas, o que também pode expô-lo a muitos comportamentos de risco.

Porém poucos estudos sobre habilidades sociais e uso de substância foram encontrados em nosso país, revelando que a produção em habilidades sociais é muito recente comparada à produção em outros países, como os de língua inglesa, que compõem o maior número, e espanhola. Corroborando essa idéia, a revisão de literatura de Murta (2005) reconhece que os estudos nacionais nessa área têm início recente, mas apresentam delineamentos pré-experimentais em contextos diversificados e cuidados metodológicos relevantes.

As pesquisas têm trazido dados preocupantes sobre o aumento do uso de substâncias psicoativas entre os adolescentes e reforça a necessidade de aprofundar a investigação nessa área. Inúmeros estudos vão ao encontro do que já se observa na prática clínica, em que muitas vezes os adolescentes abusadores e dependentes de drogas podem apresentar déficits em habilidades sociais, não conseguindo recusar a oferta de drogas para serem aceitos no grupo de iguais. Os achados de Barkin, Smith \& Durant (2002) comprovam a existência de tais déficits, principalmente quanto à dificuldade em resistir às drogas e dizer não, além de reconhecer que o desenvolvimento de habilidades de resistência ao oferecimento de drogas, auto-eficácia e tomada de decisões pode auxiliar na redução do comportamento de uso de substâncias psicoativas.

Estudar a respeito das habilidades sociais em adolescentes usuários de substâncias revelou a necessidade de novas pesquisas em nosso país envolvendo esse tema. Nesse sentido, faz-se necessária a realização de mais estudos, principalmente em populaçóes clínicas, investigando a efetividade de programas preventivos ao uso de substâncias baseados no treinamento das habilidades sociais dos adolescentes.

Porém, para que seja possível a implementação de intervençôes realmente eficientes na melhora dos déficits encontrados nesse quadro clínico, é importante, inicialmente, a compreensão do papel do desempenho social nos transtornos por uso de substâncias. Dessa forma, pode haver a possibilidade da ocorrência de mudanças comportamentais consistentes relacionadas à diminuição e cessação do consumo. 


\section{REFERÊNCIAS BIBLIOGRÁFICAS}

Alexandre, N. L.; Del Rio, M. P. \& Pol, A. P. (2004). Estrategias de afrontamiento: factores de protección en el consumo de alcohol, tabaco y cannabis. Adicciones, 16, 4, 1-6.

Aliane, P. P.; Lourenço, L. M. \& Ronzani, T. M. (2006). Estudo comparativo das habilidades sociais de dependentes e não dependentes de álcool. Psicologia em Estudo, 11, 1, 8388.

Amengual, M. M. (2000). Enfoques preventivos del uso de cannabis y problemas asociados. Adicciones, 12, 2, 281-300.

American Psychiatric Association, APA. (2002). Manual diagnóstico e estatístico de transtornos mentais. DSM-IV-TR. Porto Alegre: Artmed.

Barkin, S. L.; Smith, K. S. \& Durant, R. H. (2002). Social skills and attitudes associated with substance use behaviors among young adolescents. Journal of Adolescent Health, 30, 448-454.

Bolognini, M.; Plancherel, B.; Laget, J. \& Halfon, O. (2004). Adolescent's suicide attempts: populations at risk, vulnerability, and substance use. Substance Use \& Misuse, 38, 16511669.

Bolsoni-Silva, A. T. (2002). Habilidades sociais: breve análise da teoria e da prática à luz da análise do comportamento. Interação (Curitiba), 6, 2, 233-242.

Botvin, G. J.; Epstein, J. A.; Baker, E.; Diaz, T. \& IfillWilliams, M. (1997). School-based drug abuse prevention with inner-city minority youth. Journal of Child \& Adolescent Substance Abuse, 6, 1, 5-19.

Botvin, G. J.; Malgady, R. G.; Griffin, K. W.; Scheier, L. M. \& Epstein, J. A. (1998). Alcohol and marijuana use among rural youth: interaction of social and intrapersonal influences. Addictive Behaviors, 23, 3, 379-387.

Botvin, G. J. (2000a). Preventing alcohol and tobacco use through life skills training: theory, methods, and empirical findings. Alcohol Research and Health, 24, 4, 250-258.

Botvin, G. J. (2000b). Preventive drug abuse in schools: social and competence enhancement approaches targeting individual-level etiologic factors. Addictive Behaviors, 25, 6, 887897.

Botvin, G. J. \& Kantor, L. W. (2000). Preventing alcohol and tobacco use through life skills training. Alcohol Research \& Health, 24, 4, 250-257.

Botvin, G. J.; Griffin, K. W.; Diaz, T. \& Ifill-Williams, M. (2001). Drug abuse prevention among minority adolescents: posttest and one-year follow-up of a school-based preventive intervention. Prevention Science, 2, 1, 1-13.

Botvin, G. J. \& Griffin, K. W. (2002). Life skills training as a primary prevention approach for adolescent drug abuse and other problem behaviors. International Journal of Emergency Mental Health, 4, 1, 41-47. 
. (2004). Life skills training: empirical findings and future directions. The Journal of Primary Prevention, 25, 2, 211-232.

Brandt, C. C. J. (2003). The development of a substance abuse prevention programme for early adolescents in KwaZulu Natal. Dissertation, University of Pretoria, South Africa.

Caballo, V. E. (1998). El entrenamiento en habilidades sociales. Em Caballo, V. E. (Org.). Manual de técnicas de terapia y modificación de conducta (pp. 3-14; 403-471). Madrid: Siglo Veintiuno.

- (2003). Manual de avaliação e treinamento das habilidades sociais. São Paulo: Livraria Santos Editora.

Carlini, E. A.; Galduróz, J. C.; Noto, A. R. \& Nappo, S. A. (2002). Io Levantamento domiciliar sobre o uso de drogas psicotrópicas no Brasil. Centro Brasileiro de Informaçōes sobre Drogas Psicotrópicas. São Paulo: UNIFESP.

Denis, C.; Lávie, E.; Fatséas, M. \& Auriacombe, M. (2006). Psychotherapeutic interventions for cannabis abuse and/or dependence in outpatient settings (Protocol for a Cochrane Review). The Cochrane Library, 1. Oxford: Update Software.

Del Prette, Z. A. P. \& Del Prette, A. (1999). Psicologia das habilidades sociais: terapia e educação. Petrópolis: Vozes.

. (2002). Transtornos psicológicos e habilidades sociais. Em Guillardi, H. J. (Org.). Sobre comportamento e cognição: contribuiçôes da construção da teoria do comportamento (pp. 377-386). São Paulo: ESETec.

Del Prette, Z. A. P.; Del Prette, A.; Barreto, M. C. M.; Bandeira, M.; Rios-Saldaña, M. R.; Ulian, A. L. A. O.; Gerk-Carneiro, E. F.; Falcone, E. M. O. \& Villa, M. B. (2004). Habilidades sociais de estudantes de Psicologia: um estudo multicêntrico. Psicologia Reflexão e Crítica, 17, 3, 341-350.

Donohue, B.; Van-Hasselt, V. B.; Hersen, M. \& Perrin, S. (1999). Substance refusal skills in a population of adolescents diagnosed with conduct disorder and substance abuse. Adicctive Behavior, 24, 1, 37-46.

Eisen, M.; Zellmann, G. L. \& Murray, D. M. (2003). Evaluating the Lions-Quest "Skills for Adolescent" drug education program. Secon-year behavior outcomes. Addictive Behaviors, 28, 5, 883-897.

Faggiano, F.; Vigna-Tagliant, F. D.; Versino, E.; Zambon, A.; Borraccino, A. \& Lemma P. (2006). School-based prevention for illicit drug's use (Cochrane Review). The Cochrane Library, 1. Oxford: Update Software.

Falcone, E. M. O. (2000). Habilidades sociais: para além da assertividade. Em Wielenska, R. C. (Org.). Sobre comportamento e cognição: questionando e ampliando a teoria e as intervençôes clínicas e em outros contextos (pp. 211-221). São Paulo, ESETec.

. (2002). Contribuiçõos para o treinamento de habilidades de interação. Em Guilhardi, H. J.; Madi, M. B. B. P.; Queiroz, P. P. \& Scoz, M. C. (Orgs.). Sobre com- 
portamento e cognição: contribuições para a construção da teoria do comportamento (pp. 91-104). Santo André: ESETec.

Furtado, E. S.; Falcone, E. M. O. \& Clark, C. (2003). Avaliação do estresse e das habilidades sociais na experiência acadêmica de estudantes de medicina de uma universidade do Rio de Janeiro. Interação (Curitiba), 7, 2, 43-51.

Gates, S.; McCambridge, J.; Smith, L. A. \& Foxcroft, D. R. (2005). Interventions for prevention of drug use by young people delivered in non-school settings (Cochrane Review). The Cochrane Library, 1, Oxford: Update Software.

Goldberg-Lillehoj, C. J.; Spoth, R. \& Trudeau, L. (2005). Assertiveness among young rural adolescents: relationship to alcohol use. Journal of Child \& Adolescent Substance Abuse, 14, 3, 39-68.

Gorayeb, R.; Cunha Netto, J. R. \& Bugliani, M. A. P. (2003). Promoção de saúde na adolescência: experiência com programas de ensino de habilidades de vida. Em Trindade, Z. A. \& Andrade, N. A. (Orgs.). Psicologia e saúde: um campo em construção (pp. 89100). São Paulo: Casa do Psicólogo.

Graña Gómez, J. L. (2001). Análise dos fatores de risco e de proteção para o consumo de drogas em adolescentes. Em Marinho, M. L. \& Caballo, V. E. (Orgs.). Psicologia clínica e da saúde (pp. 55-75). Londrina: Ed. UEL.

Hecht, M. L.; Marsiglia, F. F.; Eleck, E.; Wagstaff, D. A.; Kulius, S.; Dustman, P. \& MillerDay, M. (2003). Culturally grounded substance use prevention: an evaluation of the keepin'it R.E.A.L. curriculum. Prevention Science, 4, 4, 233-248.

Herrmann, D. S. \& McWhirter, J. J. (1997). Refusal and resistance skills for children and adolescents: a selected review. Journal of Counseling and Development, 75, 3, 177-187.

Kaminer, Y. (1999). Addictive disorders in adolescents. Addictive Disorders, 22, 2, $275-$ 288.

Kaminer, Y. \& Szobot, C. (2004). O tratamento de adolescentes com transtornos por uso de substâncias psicoativas. Em Pinsky, I. \& Bessa, M. A. (Eds.). Adolescência e drogas (pp. 164-178). São Paulo: Contexto.

Löhr, S. S. (2001). Desenvolvimento das habilidades sociais como forma de prevenção. Em Guilhardi, H. C.; Madi, M. B. B. P.; Queiroz, P. P. \& Scoz, M. C. (Orgs.). Sobre comportamento e cognição: expondo a variabilidade (pp. 191-194). São Paulo: ESETec.

Marques, A. C. P. R. \& Cruz, M. (2000). O adolescente e o uso de drogas. Revista Brasileira de Psiquiatria, 22, 2, 32-36.

Minto, E. C.; Pedro, C. P.; Netto, J. R. C.; Bugliani. M. A. P. \& Gorayeb, R. (2006). Ensino de habilidades de vida na escola: uma experiência com adolescentes. Psicologia em Estudo, 11, 3, 561-568.

Murta, S. G. (2005). Aplicações do treinamento em habilidades sociais: análise da produção nacional. Psicologia Reflexão e Crítica, 18, 2, 283-291. 
Nightingale, E. O. \& Fischhoff, B. (2002). Adolescent risk and vulnerability: overview. Journal of Adolescent Health, 31, 1, 3-9.

Noto, A. R. (2004). Os índices de psicotrópicos entre adolescentes no Brasil. Em Pinsky, I. \& Bessa, M. A. (Eds.). Adolescência e drogas (pp. 45-53). São Paulo: Contexto.

O'Hearn, T. C. \& Gatz, M. (2002). Going for the goal: improving youths' problemsolving skills through a school-based intervention. Journal of Community Psychology, 30, 3, 281-303.

Oliveira, M. S. (2002). Abordagens psicoterápicas. Em Pulcherio, G.; Bicca, C. \& Silva, F. A. (Orgs.). Álcool, outras drogas, informação: o que cada profissional precisa saber (pp. 125-145). São Paulo: Casa do Psicólogo.

Organización Panamericana de la Salud. (2001). División de promoción y Protección de la Salud, Programa de Salud Familiar y Población, Unidad Técnica de Adolescencia. Enfoque de habilidades para la vida para un desarrollo saludable de niños y adolescentes. Fundación W. K. Kellog, Washington, D.C.

Payton, J. W.; Wardlaw, D. M.; Graczyk, P. A. \& Bloodworth, M. R (2000). Social and emocional learning: a framework for promoting mental health and reducing risk behavior in children and youth. Journal of School Health, 70, 5, 179-185.

Rosenbaum, D. P. \& Hanson, G. S. (1998). Assessing the effects of school-based drug education: a six-year multilevel analysis of Project D.A.R.E. Journal of Research in Crime and Delinquency, 35, 4, 381-412.

Scheier, L. \& Botvin, G. J. (1998). Relations of social skills, personal competence, and adolescent alcohol use: a developmental exploratory study. Journal of Early Adolescence, 18, 1, 77-114.

Scheier, L. M.; Botvin, G. J.; Diaz, T. \& Griffin, K. W. (1999). Social skills, competence, and drug refusal efficacy as predictors of adolescent alcohol use. Journal of Drug Education, 29, 251-78.

Scheier, L. M. (2001). Perceived neighborhood risk as a predictor of drug use among urban ethnic minority adolescents: moderating influences of psychosocial functioning. Journal of Child \& Adolescent Substance Abuse, 11, 2, 67-105.

Silva, V. M. \& Mattos, H. F. (2004). Os jovens são mais vulneráveis às drogas? Em Pinsky, I. \& Bessa, M. A. (Eds.). Adolescência e drogas (pp. 31-43). São Paulo: Contexto.

Simkin, D. R. (2002). Adolescent substance use disorders and comorbidity. Pediatric Clinics of North America, 49, 2, 463-477.

Spooner, C. (2000). Causes and correlates of adolescent drug abuse and implications for treatment. Drug and Alcohol Review, 18, 4, 453-475.

Suelves, J. M. \& Sánchez-Turet, M. (2001). Asertividad y uso de sustancias en la adolescencia: resultados de un estudio transversal. Anales de Psicología, 17, 1, 15-22.

Tavares, B. F.; Béria, J. U. \& Lima, M. S. (2001). Prevalência do uso de drogas e desempenho escolar entre adolescentes. Revista de Saúde Pública, 35, 2, 150-158. 
Tripathi, B. M.; Lal, R. \& Kumar, N. (2001). Substance abuse in children and adolescents: an overview. Journal of Personality and Clinical Studies, 17, 2, 67-74.

Trudeau, L.; Lillehoj, C.; Spoth, R. \& Redmond, C. (2003). The role of assertiveness and decision making in early adolescent substance initiation: mediating processes. Journal of Research on Adolescence, 13, 3, 301-328.

Weinberg, N. Z. (2001). Risk factors for adolescent substance abuse. Journal of Learning Disabilities, 34, 4, 343-351.

Williams, R. J. \& Chang, S. Y. (2000). A comprehensive and comparative review of adolescent substance abuse treatment outcome. Clinical Psychology: Science and Practice, 7, 2, 138-166.

\section{NoTAS}

1 Artigo baseado na Dissertação de Mestrado em Psicologia Clínica, intitulada Habilidades sociais em adolescentes usuários de maconha, de autoria de Marcia Fortes Wagner, defendida pelo Programa de Pós-Graduação em Psicologia da Pontifícia Universidade Católica do Rio Grande do Sul (PUCRS), com apoio CNPq.

\section{AneXo}

\section{TABELA 1}

Distribuição percentual das publicações localizadas nos sistemas de banco de dados computadorizados:

\begin{tabular}{|lcc|}
\hline Sistemas & $\begin{array}{c}\text { Artigos } \\
\mathbf{n}\end{array}$ & \multicolumn{1}{c|}{$\%$} \\
\hline MEDLINE & 10 & 20,41 \\
WEB-OF-SCIENCE & 11 & 22,45 \\
PROQUEST & 06 & 12,24 \\
PSYCINFO & 12 & 24,49 \\
LILACS & 06 & 12,24 \\
COCHRANELIBRARY & 04 & 8,16 \\
TOTAL & 49 & 100,00 \\
\hline
\end{tabular}

Recebido em 8 de setembro de 2007 Aceito para publicação em $1^{\circ}$ de dezembro de 2007 